\title{
ON THE GEOMETRIC MEANS OF ENTIRE FUNCTIONS OF SEVERAL COMPLEX VARIABLES $\left({ }^{1}\right)$
}

\author{
BY \\ A. K. AGARWAL $\left({ }^{2}\right)$
}

\begin{abstract}
Let $f\left(z_{1}, \ldots, z_{n}\right)$ be an entire function of the $n(\geqq 2)$ complex variables $z_{1}, \ldots, z_{n}$, holomorphic for $\left|z_{t}\right| \leqq r_{t}, t=1, \ldots, n$. We have considered the case of only two complex variables for simplicity. Recently many authors have defined the arithmetic means of the function $\left|f\left(z_{1}, z_{2}\right)\right|$ and have investigated their properties. In the present paper, the geometric means of the function $\left|f\left(z_{1}, z_{2}\right)\right|$ have been defined and the asymptotic behavior of certain growth indicators for entire functions of several complex variables have been studied and the results are given in the form of theorems.
\end{abstract}

1. Let

$$
f\left(z_{1}, \ldots, z_{n}\right)=\sum_{k_{1}, \ldots, k_{n} \geqq 0} a_{k_{1}} \ldots k_{n} z_{1}^{k_{1}} \cdots z_{n^{n}}^{k_{n}}
$$

be an entire function of the $n(\geqq 2)$ complex variables $z_{1}, \ldots, z_{n}$, holomorphic for $\left|z_{t}\right| \leqq r_{t}, t=1, \ldots, n$. Let us denote the maximum modulus of the function $f\left(z_{1}, \ldots, z_{n}\right)$ as

$$
M\left(r_{1}, \ldots, r_{n}\right)=\max _{\left|z_{t}\right| \leqq r_{t}}\left|f\left(z_{1}, \ldots, z_{n}\right)\right| \quad(t=1, \ldots, n) .
$$

Here we consider the case of only two complex variables for simplicity. The results can easily be extended to several complex variables.

The geometric mean of $\left|f\left(z_{1}, z_{2}\right)\right|$ for $\left|z_{t}\right| \leqq r_{t}(t=1,2)$ has been defined as [4]

$$
G\left(r_{1}, r_{2}\right)=\exp \left\{\frac{1}{(2 \pi)^{2}} \int_{0}^{2 \pi} \int_{0}^{2 \pi} \log \left|f\left(r_{1} e^{i \theta_{1}}, r_{2} e^{i \theta_{2}}\right)\right| d \theta_{1} d \theta_{2}\right\}
$$

Further, let us define

$$
g_{k}\left(r_{1}, r_{2}\right)=\exp \left\{\frac{(k+1)^{2}}{\left(r_{1} r_{2}\right)^{k+1}} \int_{0}^{r_{1}} \int_{0}^{r_{2}}\left(x_{1} x_{2}\right)^{k} \log G\left(x_{1}, x_{2}\right) d x_{1} d x_{2}\right\},
$$

where $k$ is any positive number.

Received by the editors September 20, 1968 and, in revised form, March 25, 1970.

AMS 1969 Subject Classifications. Primary 3205, 3210; Secondary 3217.

Key Words and Phrases. Entire function, holomorphic, geometric means, order, lower order, Poisson formula in two variables, slowly changing function, regular growth.

( ${ }^{1}$ ) This research has been supported by the Senior Research Fellowship award of the CSIR, New Delhi, India.

(2) Present address: Department of Mathematics, Grambling College, Grambling, Louisiana 71245. 
The finite order $\rho$ of an entire function $f\left(z_{1}, z_{2}\right)$ is defined as [2, p. 219]

$$
\limsup _{r_{1}, r_{2} \rightarrow \infty} \frac{\log \log M\left(r_{1}, r_{2}\right)}{\log \left(r_{1} r_{2}\right)}=\rho .
$$

Similarly, we can define the lower order $\lambda$ as

$$
\liminf _{r_{1}, r_{2} \rightarrow \infty} \frac{\log \log M\left(r_{1}, r_{2}\right)}{\log \left(r_{1} r_{2}\right)}=\lambda,
$$

where $\lambda=\min \left(\lambda_{1}, \lambda_{2}\right)$ and

$$
\begin{aligned}
& \lambda_{1}=\liminf _{r_{2} \rightarrow \infty} \liminf _{r_{1} \rightarrow \infty} \frac{\log \log M\left(r_{1}, r_{2}\right)}{\log \left(r_{1} r_{2}\right)}, \\
& \lambda_{2}=\liminf _{r_{1} \rightarrow \infty} \liminf _{r_{2} \rightarrow \infty} \frac{\log \log M\left(r_{1}, r_{2}\right) .}{\log \left(r_{1} r_{2}\right)} .
\end{aligned}
$$

In this paper we have investigated a few properties of the above defined mean values $G\left(r_{1}, r_{2}\right)$ and $g_{k}\left(r_{1}, r_{2}\right)$; the results are given in the form of theorems.

2. THEOREM 1. Let $f\left(z_{1}, z_{2}\right)$ be an entire function of finite order $\rho$ and lower order $\lambda$ (nonintegral with respect to $z_{1}$ and $z_{2}$ ), then

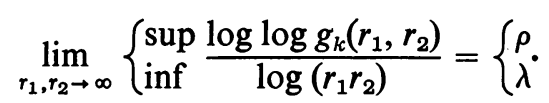

Proof. From (1.1), it follows that $G\left(r_{1}, r_{2}\right)$ is an increasing function of (i) $r_{1}$ for given $r_{2}$, (ii) $r_{2}$ for a given $r_{1}$, and (iii) $r_{1}$ and $r_{2}$ both increasing.

Next from (1.1) we have

$$
\int_{0}^{r_{1}} \int_{0}^{r_{2}}\left(x_{1} x_{2}\right)^{k} \log G\left(x_{1}, x_{2}\right) d x_{1} d x_{2} \leqq \frac{\left(r_{1} r_{2}\right)^{k+1}}{(k+1)^{2}} \log M\left(r_{1}, r_{2}\right) .
$$

Hence

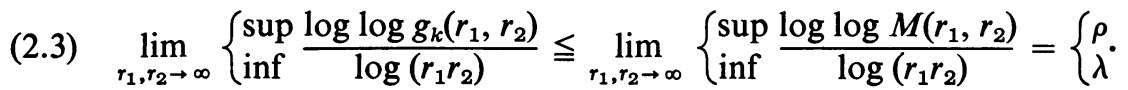

Further, if $f\left(z_{1}, z_{2}\right)$ is analytic in $\left|z_{1}\right| \leqq R_{1},\left|z_{2}\right| \leqq R_{2}, z_{j}=r_{j} e^{i \theta_{j}}$ and $\zeta_{j}=R_{j} e^{i \phi_{j}}$ $(j=1,2)$, then

$$
\log \left|f\left(z_{1}, z_{2}\right)\right| \leqq \int_{0}^{2 \pi} \int_{0}^{2 \pi} P\left(z_{1}, \zeta_{1}\right) P\left(z_{2}, \zeta_{2}\right) \log \left|f\left(\zeta_{1}, \zeta_{2}\right)\right| d \phi_{1} d \phi_{2}
$$

where

$$
P\left(z_{s}, \zeta_{s}\right)=\frac{1}{2 \pi}\left\{\frac{R_{s}^{2}-r_{s}^{2}}{R_{s}^{2}-2 r_{s} R_{s} \cos \left(\phi_{s}-\theta_{s}\right)+r_{s}^{2}}\right\} \quad(s=1,2)
$$

Using (2.4) for an entire function $f\left(z_{1}, z_{2}\right)$, we get

$$
\log \left|f\left(r_{1} e^{i \theta_{1}}, r_{2} e^{i \theta_{2}}\right)\right| \leqq \frac{R_{1}+r_{1}}{R_{1}-r_{1}} \frac{R_{2}+r_{2}}{R_{2}-r_{2}} \log G\left(R_{1}, R_{2}\right) .
$$


Taking $R_{1}=\alpha r_{1}, R_{2}=\alpha r_{2}(\alpha>1)$, we have from (2.5)

$$
\log M\left(r_{1}, r_{2}\right) \leqq\{(\alpha+1) /(\alpha-1)\}^{2} \log G\left(\alpha r_{1}, \alpha r_{2}\right) .
$$

Therefore

$$
\begin{aligned}
\log g_{k}\left(\alpha r_{1}, \alpha r_{2}\right) & \geqq\{(\alpha-1) /(\alpha+1)\}^{2}\left\{1-1 / \alpha^{k+1}\right\}^{2} \log M\left(r_{1} / \alpha, r_{2} / \alpha\right) \\
& =H\left\{\log M\left(r_{1} / \alpha, r_{2} / \alpha\right)\right\},
\end{aligned}
$$

where $H>0$ and is independent of $r_{1}$ and $r_{2}$. Hence

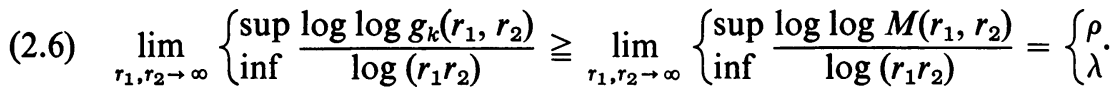

Combining (2.3) and (2.6), we get (2.1).

3. Let $\phi\left(r_{1}, r_{2}\right)$ be a "slowly changing" function; that is, $\phi\left(r_{1}, r_{2}\right)>0$ and is continuous for $r_{1}>r_{1}^{0}, r_{2}>r_{2}^{0}$ and for every constant $l, m>0, \phi\left(l r_{1}, m r_{2}\right) \sim \phi\left(r_{1}, r_{2}\right)$ as $r_{1}$ or $r_{2}$, or $r_{1}$ and $r_{2}$, tend to infinity.

Also let

$$
\lim _{r_{1}, r_{2} \rightarrow \infty}\left\{\begin{array}{l}
\sup \\
\inf \frac{\log g_{k}\left(r_{1}, r_{2}\right)}{\left(r_{1} r_{2}\right)^{\rho} \phi\left(r_{1}, r_{2}\right)}=\left\{\begin{array}{l}
p \\
q
\end{array} \quad(0<q \leqq p<\infty)\right.
\end{array}\right.
$$

and

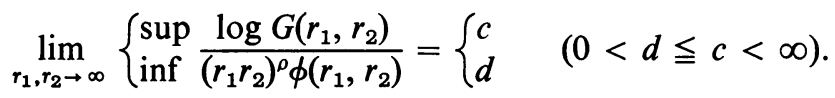

THEOREM 2. If $f\left(z_{1}, z_{2}\right)$ be an entire function of finite nonzero order $\rho$, then

$$
\text { (3.5) } c+\left\{\rho(2 k+3 \rho+2) /(k+\rho+1)^{2}\right\} d \leqq p\{1+\rho /(k+1)\}^{2}\{1+(k+1) / \rho\}^{2 \rho /(k+1)} \text {. }
$$

Proof. For $0<\eta<1$, from (1.2) we have

(3.6) $\log g_{k}\left(r_{1}+\eta r_{1}, r_{2}+\eta r_{2}\right)$

$$
\begin{aligned}
= & \frac{(k+1)^{2}}{\left(r_{1} r_{2}\right)^{k+1}(1+\eta)^{2(k+1)}} \int_{0}^{r_{1}+\eta r_{1}} \int_{0}^{r_{2}+\eta r_{2}}\left(x_{1} x_{2}\right)^{k} \log G\left(x_{1}, x_{2}\right) d x_{1} d x_{2} \\
< & \frac{A}{\left(r_{1} r_{2}\right)^{k+1}}+\left(\frac{r_{1}^{0}}{r_{1}}\right)^{k+1}\left\{\frac{\log G\left(r_{1}^{0}, r_{2}\right)}{(1+\eta)^{2(k+1)}}+\frac{\left((1+\eta)^{k+1}-1\right) \log G\left(r_{1}^{0}, r_{2}+\eta r^{2}\right)}{(1+\eta)^{2(k+1)}}\right\} \\
& +\left(\frac{r_{2}^{0}}{r_{2}}\right)^{k+1}\left\{\frac{\log G\left(r_{1}, r_{2}^{0}\right)}{(1+\eta)^{2(k+1)}}+\frac{\left((1+\eta)^{k+1}-1\right) \log G\left(r_{1}+\eta r_{1}, r_{2}^{0}\right)}{(1+\eta)^{2(k+1)}}\right\} \\
& +\frac{(k+1)\left((1+\eta)^{k+1}-1\right)}{(1+\eta)^{2(k+1)}}\left\{\frac{1}{r_{1}^{k+1}} \int_{r_{1}^{0}}^{r_{1}} x_{1}^{k} \log G\left(x_{1}, r_{2}+\eta r_{2}\right) d x_{1}\right. \\
& \left.+\frac{1}{r_{2}^{k}+1} \int_{r_{2}^{0}}^{r_{2}} x_{2}^{k} \log G\left(r_{1}+\eta r_{1}, x_{2}\right) d x_{2}\right\}
\end{aligned}
$$




$$
\begin{aligned}
& +\frac{(c+\varepsilon)(k+1)^{2}}{\left(r_{1} r_{2}\right)^{k+1}(1+\eta)^{2(k+1)}} \int_{r_{1}^{0}}^{r_{1}} \int_{r_{2}^{0}}^{r_{2}}\left(x_{1} x_{2}\right)^{k+\rho} \phi\left(x_{1}, x_{2}\right) d x_{1} d x_{2} \\
& +\frac{\left((1+\eta)^{k+1}-1\right)^{2}}{(1+\eta)^{2(k+1)}} \log G\left(r_{1}+\eta r_{1}, r_{2}+\eta r_{2}\right)
\end{aligned}
$$

where $A$ is a constant,

$$
\begin{aligned}
\sim & \frac{A}{\left(r_{1} r_{2}\right)^{k+1}}+\left(\frac{r_{1}^{0}}{r_{1}}\right)^{k+1}\left\{\frac{\log G\left(r_{1}^{0}, r_{2}\right)}{(1+\eta)^{2(k+1)}}+\frac{\left((1+\eta)^{k+1}-1\right) \log G\left(r_{1}^{0}, r_{2}+\eta r_{2}\right)}{(1+\eta)^{2(k+1)}}\right\} \\
& +\left(\frac{r_{2}^{0}}{r_{2}}\right)^{k+1}\left\{\frac{\log G\left(r_{1}, r_{2}^{0}\right)}{(1+\eta)^{2(k+1)}}+\frac{\left((1+\eta)^{k+1}-1\right) \log G\left(r_{1}+\eta r_{1}, r_{2}^{0}\right)}{(1+\eta)^{2(k+1)}}\right\} \\
& +\left\{\frac{k+1}{k+\rho+1}\right\} \frac{(c+\varepsilon)\left(r_{1} r_{2}\right)^{\rho}\left((1+\eta)^{k+1}-1\right)}{(1+\eta)^{2(k+1)}} \cdot\left\{\phi\left(r_{1}, r_{2}+\eta r_{2}\right)+\phi\left(r_{1}+\eta r_{1}, r_{2}\right)\right\} \\
& +\left\{\frac{k+1}{k+\rho+1}\right\}^{2} \frac{(c+\varepsilon)\left(r_{1} r_{2}\right)^{\rho} \phi\left(r_{1}, r_{2}\right)}{(1+\eta)^{2(k+1)}} \\
& +\frac{\left((1+\eta)^{k+1}-1\right)^{2}}{(1+\eta)^{2(k+1)}} \log G\left(r_{1}+\eta r_{1}, r_{2}+\eta r_{2}\right)
\end{aligned}
$$

from [3, Lemma 5].

Hence, dividing both the sides by $\left\{\left(r_{1}+\eta r_{1}\right)^{\rho}\left(r_{2}+\eta r_{2}\right)^{\rho} \phi\left(r_{1}+\eta r_{1}, r_{2}+\eta r_{2}\right)\right\}$ and taking limit, we get

$$
\begin{aligned}
\limsup _{r_{1}, r_{2} \rightarrow \infty}\left\{\frac{\log g_{k}\left(r_{1}, r_{2}\right)}{\left(r_{1} r_{2}\right)^{\rho} \phi\left(r_{1}, r_{2}\right)}\right\} \leqq c[ & \left\{\frac{k+1}{k+\rho+1}\right\}^{2} \frac{1}{(1+\eta)^{2(k+\rho+1)}} \\
& \left.+2\left\{\frac{k+1}{k+\rho+1}\right\} \frac{\left((1+\eta)^{k+1}-1\right)}{(1+\eta)^{2(k+\rho+1)}}+\frac{\left((1+\eta)^{k+1}-1\right)^{2}}{(1+\eta)^{2(k+1)}}\right] .
\end{aligned}
$$

Since $\eta$ is arbitrary, we get

$$
p \leqq\{(k+1) /(k+\rho+1)\}^{2} c .
$$

Next, from (3.6), we have

(3.11) $\log g_{k}\left(r_{1}+\eta r_{1}, r_{2}+\eta r_{2}\right)$

$$
\begin{aligned}
& >\frac{(d-\varepsilon)(k+1)^{2}}{\left(r_{1} r_{2}\right)^{k+1}(1+\eta)^{2(k+1)}} \int_{r_{1}^{0}}^{r_{1}} \int_{r_{2}^{0}}^{r_{2}}\left(x_{1} x_{2}\right)^{k+\rho} \phi\left(x_{1}, x_{2}\right) d x_{1} d x_{2} \\
& +\frac{(d-\varepsilon)(k+1)\left((1+\eta)^{k+1}-1\right)}{(1+\eta)^{2(k+1)}}\left\{\frac{r_{2}^{\rho}}{r_{1}^{k+1}} \int_{r_{1}^{0}}^{r_{1}} x_{1}^{k+\rho} \phi\left(x_{1}, r_{2}\right) d x_{1}\right. \\
& \left.+\frac{r_{1}^{\rho}}{r_{2}^{k+1}} \int_{r_{2}^{0}}^{r_{2}} x_{2}^{k+\rho} \phi\left(r_{1}, x_{2}\right) d x_{2}\right\} \\
& \quad+\frac{\left((1+\eta)^{k+1}-1\right)^{2}}{(1+\eta)^{2(k+1)}} \log G\left(r_{1}, r_{2}\right)
\end{aligned}
$$




$$
\begin{aligned}
& \sim \frac{(d-\varepsilon)\left(r_{1} r_{2}\right)^{\rho} \phi\left(r_{1}, r_{2}\right)}{(1+\eta)^{2(k+1)}}\left[\left\{\frac{k+1}{k+\rho+1}\right\}^{2}+2\left\{\frac{k+1}{k+\rho+1}\right\}\left\{(1+\eta)^{k+1}-1\right\}\right] \\
& \quad+\left[\frac{\left\{(1+\eta)^{k+1}-1\right\}}{(1+\eta)^{2(k+1)}}\right] \log G\left(r_{1}, r_{2}\right),
\end{aligned}
$$

using [3, Lemma 5].

Hence

$$
q \geqq \frac{d}{(1+\eta)^{2(k+\rho+1)}}\left[\left\{\frac{k+1}{k+\rho+1}\right\}+\left\{(1+\eta)^{k+1}-1\right\}\right]^{2} .
$$

Since $\eta$ is arbitrary, we get

$$
q \geqq\{(k+1) /(k+\rho+1)\}^{2} d .
$$

Combining (3.10) and (3.13), the inequality (3.3) follows. Also, from (3.11)

$$
\left\{(1+\eta)^{k+1}-1\right\}^{2} c \leqq p(1+\eta)^{2(k+\rho+1)}-d\left[\left\{\frac{k+1}{k+\rho+1}\right\}^{2}+2\left\{\frac{k+1}{k+\rho+1}\right\}\left\{(1+\eta)^{k+1}-1\right\}\right]
$$

and so for all $\eta>0$

$$
c \leqq p(1+\eta)^{2(k+\rho+1)} /\left\{(1+\eta)^{k+1}-1\right\}^{2} .
$$

The right-hand side of this inequality has the least value when $(1+\eta)^{k+1}=$ $\{1+(k+1) / \rho\}$, therefore

$$
c \leqq p\{1+\rho /(k+1)\}^{2}\{1+(k+1) / \rho\}^{2 \rho /(k+1)} .
$$

Also, from (3.14) we get

$$
c+\frac{\rho(2 k+3 \rho+2)}{(k+\rho+1)^{2}} d \leqq p\{1+\rho /(k+1)\}^{2}\{1+(k+1) / \rho\}^{2 \rho /(k+1)},
$$

which completes the proof of the theorem.

4. THEOREM 3. Let $f\left(z_{1}, z_{2}\right)$ be an entire function of finite order $\rho$ and if

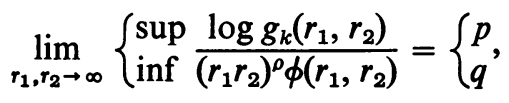

and

$$
\log G\left(r_{1}, r_{2}\right) \sim \beta\left(r_{1} r_{2}\right)^{\rho} \phi\left(r_{1}, r_{2}\right),
$$

for large values of $r_{1}$ and $r_{2}$, where $\beta$ is a constant and $0<q \leqq p<\infty$ and $\phi\left(r_{1}, r_{2}\right)$ as defined in \$3. Then

(i) $f\left(z_{1}, z_{2}\right)$ is of regular growth;

(ii) $(k+\rho+1)^{2} p=(k+1)^{2} \beta=(k+\rho+1)^{2} q$; and

(iii) $\lim _{r_{1}, r_{2} \rightarrow \infty} \log g_{k}\left(r_{1}, r_{2}\right) / \log G\left(r_{1}, r_{2}\right)=\{(k+1) /(k+\rho+1)\}^{2}$.

Proof. (i) Taking the logarithm of (4.2), the result follows. 
(ii) From (1.2), we have

(4.3) $\log g_{k}\left(r_{1}, r_{2}\right)<\frac{A}{\left(r_{1} r_{2}\right)^{k+1}}+\left\{\frac{r_{1}^{0}}{r_{1}}\right\}^{k+1} \log G\left(r_{1}^{0}, r_{2}\right)+\left\{\frac{r_{2}^{0}}{r_{2}}\right\}^{k+1} \log G\left(r_{1}, r_{2}^{0}\right)$

$$
+\frac{\beta(k+1)^{2}}{\left(r_{1} r\right)^{k+1}} \int_{r_{1}^{0}}^{r_{1}} \int_{r_{2}^{0}}^{r_{2}}\left(x_{1} x_{2}\right)^{k+\rho} \phi\left(x_{1}, x_{2}\right) d x_{1} d x_{2}
$$

from (4.2) and $A$ is a constant,

$$
\begin{aligned}
& \sim \frac{A}{\left(r_{1} r_{2}\right)^{k+1}}+\left\{\frac{r_{1}^{0}}{r_{1}}\right\}^{k+1} \log G\left(r_{1}^{0}, r_{2}\right)+\left\{\frac{r_{2}^{0}}{r_{2}}\right\}^{k+1} \log G\left(r_{1}, r_{2}^{0}\right) \\
& \quad+\beta\left\{\frac{k+1}{k+\rho+1}\right\}^{2}\left(r_{1} r_{2}\right)^{\rho} \phi\left(r_{1}, r_{2}\right)
\end{aligned}
$$

by repeated application of [3, Lemma 5].

Taking the limit, we get

$$
\lim _{r_{1}, r_{2} \rightarrow \infty} \frac{\log g_{k}\left(r_{1}, r_{2}\right)}{\left(r_{1} r_{2}\right)^{\rho} \phi\left(r_{1}, r_{2}\right)} \leqq \beta\left\{\frac{k+1}{k+\rho+1}\right\}^{2} .
$$

Also, from (1.2), we have

$$
\begin{aligned}
\log g_{k}\left(r_{1}, r_{2}\right) & >\frac{(k+1)^{2}}{\left(r_{1} r_{2}\right)^{k+1}} \int_{r_{1}^{0}}^{r_{1}} \int_{r_{2}^{0}}^{r_{2}}\left(x_{1} x_{2}\right)^{k} \log G\left(x_{1}, x_{2}\right) d x_{1} d x_{2} \\
& \sim \beta\left\{\frac{k+1}{k+\rho+1}\right\}^{2}\left(r_{1} r_{2}\right)^{\circ} \phi\left(r_{1}, r_{2}\right) \text { from (4.2) }
\end{aligned}
$$

Hence

$$
\lim _{r_{1}, r_{2} \rightarrow \infty} \frac{\log g_{k}\left(r_{1}, r_{2}\right)}{\left(r_{1} r_{2}\right)^{\rho} \phi\left(r_{1}, r_{2}\right)} \geqq \beta\left\{\frac{k+1}{k+\rho+1}\right\}^{2} .
$$

Combining (4.4) and (4.6), the result follows.

(iii) Further, if we divide by $\log G\left(r_{1}, r_{2}\right)$, a positive increasing function, the inequalities (4.3) and (4.5) respectively, we get

and

$$
\begin{aligned}
\frac{\log g_{k}\left(r_{1}, r_{2}\right)}{\log G\left(r_{1}, r_{2}\right)}< & \frac{1}{\log G\left(r_{1}, r_{2}\right)}\left[\frac{A}{\left(r_{1} r_{2}\right)^{k+1}}+\left\{\frac{r_{1}^{0}}{r_{1}}\right\}^{k+1} \log G\left(r_{1}^{0}, r_{2}\right)\right. \\
& \left.+\left\{\frac{r_{2}^{0}}{r_{2}}\right\}^{k+1} \log G\left(r_{1}, r_{2}^{0}\right)\right] \\
& +\beta\left\{\frac{k+1}{k+\rho+1}\right\}^{2} \frac{\left(r_{1} r_{2}\right)^{\circ} \phi\left(r_{1}, r_{2}\right)}{\log G\left(r_{1}, r_{2}\right)}
\end{aligned}
$$

$$
\frac{\log g_{k}\left(r_{1}, r_{2}\right)}{\log G\left(r_{1}, r_{2}\right)}>\beta\left\{\frac{k+1}{k+\rho+1}\right\}^{2} \frac{\left(r_{1} r_{2}\right)^{\rho} \phi\left(r_{1}, r_{2}\right)}{\log G\left(r_{1}, r_{2}\right)}
$$

Since (4.2) holds, on proceeding to limits, the inequalities (4.7) and (4.8) lead to the result.

In conclusion I offer my grateful thanks to Dr. S. K. Bose for his kind help in the preparation of this paper. 


\section{REFERENCES}

1. S. Bochner and W. T. Martin, Several complex variables, Princeton Math. Series, vol. 10, Princeton Univ. Press, Princeton, N. J., 1948. MR 10, 366.

2. S. K. Bose and D. Sharma, Integral functions of two complex variables, Compositio Math. 15 (1963), 210-226. MR 29 \#270.

3. G. H. Hardy and W. W. Rogosinski, Notes on Fourier series. III: Asymptotic formulae for the sums of certain trigonometrical series, Quart. J. Math. Oxford Ser. 16 (1945), 49-58. MR 7, 247.

4. R. K. Srivastava, Integral functions represented by Dirichlet series and integral functions of several complex variables, Ph.D. Thesis, Lucknow Univ., Lucknow, India, 1964.

\section{LUCKNOW UNIVERSITY,}

LUCKNOW, U. P., INDIA 